\title{
Análisis comparativo de índices bióticos utilizados en la evaluación de la calidad de las aguas en un río mediterráneo de Chile: río Chillán, VIII Región
}

\author{
Comparative analysis of biotic indexes used to evaluate water quality in a \\ Mediterranean river of Chile: Chillán River, VIII Region
}

RICARDO FIGUEROA ${ }^{1, *}$, ALEJANDRO PALMA ${ }^{2}$, VICTOR RUIZ $^{2} \&$ XAVIER NIELL ${ }^{3}$

\footnotetext{
${ }^{1}$ Centro de Ciencias Ambientales EULA-Chile, Universidad de Concepción, Casilla 160-C, Concepción, Chile ${ }^{2}$ Departamento de Zoología, Facultad de Ciencias Naturales y Oceanográficas, Universidad de Concepción, Chile ${ }^{3}$ Departamento de Ecología, Facultad de Ciencias, Universidad de Málaga, Málaga, España

*e-mail para correspondencia: rfiguero@udec.cl
}

\begin{abstract}
RESUMEN
El uso de macroinvertebrados bentónicos como indicadores biológicos es de larga tradición en los países desarrollados y son incorporados en todas las evaluaciones de calidad ecológica de sistemas fluviales. En América Latina estos estudios son menos frecuentes y las normativas para la protección de los recursos acuáticos recién comienzan a elaborarse, como es el caso de Chile, dejando abierta la posibilidad al uso de criterios biológicos. El presente estudio realiza una adaptación de índices bióticos (IBE, BMWP, IBF y SIGNAL) los que son aplicados y comparados en una cuenca mediterránea de Chile, el río Chillán. Los resultados muestran el mismo patrón de comportamiento para todos los índices, definiendo áreas en buen estado y otras fuertemente impactadas desde el punto de vista biológico. Sin embargo, el IBF y el SIGNAL son más sensibles a perturbaciones no detectadas por el IBE y BMWP. Se discute la factibilidad de aplicación y ventajas que ofrece cada uno de los índices, permitiendo hacer una propuesta metodológica para Chile central.
\end{abstract}

Palabras clave: índices bióticos, macroinvertebrados bentónicos, Chile mediterráneo.

\begin{abstract}
Developed countries have traditionally used benthic macroinvertebrates as biological indicators, incorporating them into the environmental quality evaluations of fluvial systems. These studies are less frequent in Latin America, where the environmental protection standards for aquatic resources are just beginning to be elaborated. Thus, in Chile, the use of biocriteria for such studies remains a possibility. This study of the Chillán River (Chile) adapts these biotic indexes (IBE, BMWP, IBF, and SIGNAL) for their application and comparison in a Mediterranean basin. The results showed the same behavior pattern for all indexes, defining some areas as having good conditions and others, which, from a biological perspective, have been strongly impacted. Specifically, the IBF and SIGNAL indexes are more sensitive to disturbances than the IBE and BMWP. Application feasibility and the advantages of the different indices are discussed, proposing a new methodology for central Chile.
\end{abstract}

Key words: biotic indexes, benthic macroinvertebrates, mediterranean Chile.

\section{INTRODUCCIÓN}

La mayoría de las zonas con climas mediterráneos están localizadas en la cuenca del mar Mediterráneo $\left(30-45^{\circ} \mathrm{N}\right)$ entre Europa, Asia y Africa. Las otras se encuentran en las costas del Pacífico desde el suroeste de Oregon al noroeste de Baja California $\left(31-41^{\circ} \mathrm{N}\right)$, parte del suroeste de Australia (32-38 S), suroeste de Región del Cabo en Sudáfrica $\left(32-35^{\circ} \mathrm{S}\right)$ y las costas de Chile central (32-41 $\mathrm{S}$ ) (Di Castri 1981, Strahler \& Strahler 1989). En estas zonas los ríos y arroyos son ejemplos de convergencia en términos de estructura y características funcionales, siendo Chile la más desconocida (Gasith \& Resh 1999). 
Como en las otras partes del mundo, la zona mediterránea en Chile es una de las más pobladas y de intensa actividades agrícolas e industriales, que ejercen una fuerte presión sobre el uso del suelo y el recurso hídrico. Muchas de las normativas para controlar estos impactos son de reciente elaboración en el país y aplicadas de manera experimental. Solo en los últimos años se encuentra en desarrollo la primera "Norma para la Protección de la Calidad de las Aguas Superficiales", hoy reconocida como un documento guía de la autoridad ambiental (CONAMA 2004), lo que sin duda es un paso importante como inicio en la protección de los recursos acuáticos chilenos, y cuyo objetivo central es "la protección y conservación de las comunidades acuáticas". Sin embargo, se fundamenta en valores límites de variables fisico-químicas, dejando abierta la posibilidad para abordar el problema a través de la evaluación de las comunidades biológicas.

Este tipo de enfoque ya ha sido abordado en Norteamérica (Plaftkin et al. 1989, Resh et al. 1995, 1996, Barbour 1997), Australia (Chessman 1995, 2003, Schofield \& Davies 1996, Mitzeling et al. 2002, Tiller \& Metzeling 2002), Inglaterra (Wrigth et al. 1984, Wrigth et al. 2000) África (Chuter 1972) y en general, por varios países de Europa que hoy se encuentran ajustando metodologías dentro de la Directiva Marco del Agua (Bonada 2003), siendo los macroinvertebrados bentónicos el grupo más utilizado en este tipo de estudio (Bonada et al. 2006). Especialmente por su probada sensibilidad y diversidad, que permite responder diferencialmente a varios tipos de perturbaciones y contaminantes, reflejando el efecto integrado de todas las variables ambientales (Bunn \& Davies 2000, Tiller \& Metzelling 2002, Allan 2004). Por lo tanto, reflejan la calidad integral del ecosistema (Karr \& Chu 2002) entendida como una comunidad de organismos con una composición específica, diversidad y organización funcional natural de una región conocida (Karr \& Dudley 1981, Norris \& Hawkins 2000, Simon 2000, Butcher et al. 2003) que previamente definida, puede ser utilizada como de referencia (Norris \& Thoms 1999).

En contraste a los estudios señalados, los ecosistemas acuáticos continentales chilenos han sido abordados de manera general en la últimas décadas (e.g., Campos et al. 1984, Arenas 1995, Habit et al. 1998, Figueroa et al. 2000, Figueroa et al. 2006) y solo se conoce una aplicación experimental del Índice Biótico de Familias de Hinselhoff (1988) realizada por Figueroa et al. (2003), dejando ver la escasa experiencia que se tiene en Chile en el uso de criterios biológicos, lo que amerita la necesidad de recopilar información y la estandarización de métodos de evaluación y monitoreo. Este estudio realiza una caracterización de la calidad ecológica de la cuenca del río Chillán, Chile central, mediante la comparación de índices bióticos que han sido aplicados en regiones biogeográficas similares y de amplia aceptabilidad en la comunidad científica internacional.

\section{MATERIALES Y MÉTODOS}

\section{Área de estudio}

El área de estudio comprende la cuenca hidrográfica del río Chillán, en la provincia de Ñuble, VIII Región, Chile central (36 $33^{\circ}$ - $36^{\circ} 53^{\prime}$ $\mathrm{S}, 72^{\circ} 21^{\prime}-71^{\circ} 24^{\prime}$ O) con un área de drenaje de ca. $757,7 \mathrm{~km}^{2}$. Se extiende desde los Nevados de Chillán, hasta el Valle Central, entre los 3.200 y los $75 \mathrm{~m}$ de altitud, con una longitud aproximada de $105 \mathrm{~km}$, desemboca sus aguas al río Ñuble. La parte superior de la cuenca se localiza dentro de la cordillera de los Andes con una cobertura característica de hielo y nieve, bosque nativo y escasas plantaciones forestales, que se extiende hasta el sector de Esperanza, a $570 \mathrm{~m}$ de altitud, donde el río se estrecha y da inicio a la parte de menor pendiente. Las partes media y baja están localizadas en el valle central donde predomina la actividad agrícola (remolacha, trigo, cereales, viñedos y frutales) y las actividades forestales. El clima es mediterráneo, con un periodo estival de casi cinco meses de duración (noviembremarzo). La parte media y baja de la cuenca presenta temperaturas máximas medias de $28{ }^{\circ} \mathrm{C}$ durante el mes de enero, siendo este el mes más cálido. La temperatura mínima media es en julio de ca. 3,5 ${ }^{\circ} \mathrm{C}$. Durante el periodo invernal (junioagosto) se concentran más del $50 \%$ de las precipitaciones, con un promedio de $1.025 \mathrm{~mm}$ por año. El río Chillán tiene un caudal promedio en su desembocadura de $22,9 \mathrm{~m}^{3}$, sin embargo, existen fuertes variaciones y puede llegar a valores inferiores a $1 \mathrm{~m}^{3}$ durante la época estival. Estas mínimas de caudal no es una condición natural, puesto que ocurren 
principalmente por la extracción del agua para fines de riego y secundariamente, para provisión de agua potable (Debels et al. 2005). Estos mismos autores señalan que sobre el seguimiento anual de 10 variables físicoquímicas, es posible definir que las aguas del río Chillán son de buena calidad, a excepción de las estaciones aguas abajo, que reciben el impacto de las aglomeraciones urbanas.
Este estudio consideró 18 estaciones de muestreo (Fig. 1), ocho en el curso principal (E1-E8) y 10 en los ríos tributarios (T1-T10). La estación El se sitúa en el sector de Esperanza, que cierra la parte alta de la cuenca y que de acuerdo a sus características de uso, no sería influida por algún tipo de actividad que modifique la calidad de las aguas, por lo cual se consideró como estación de referencia.
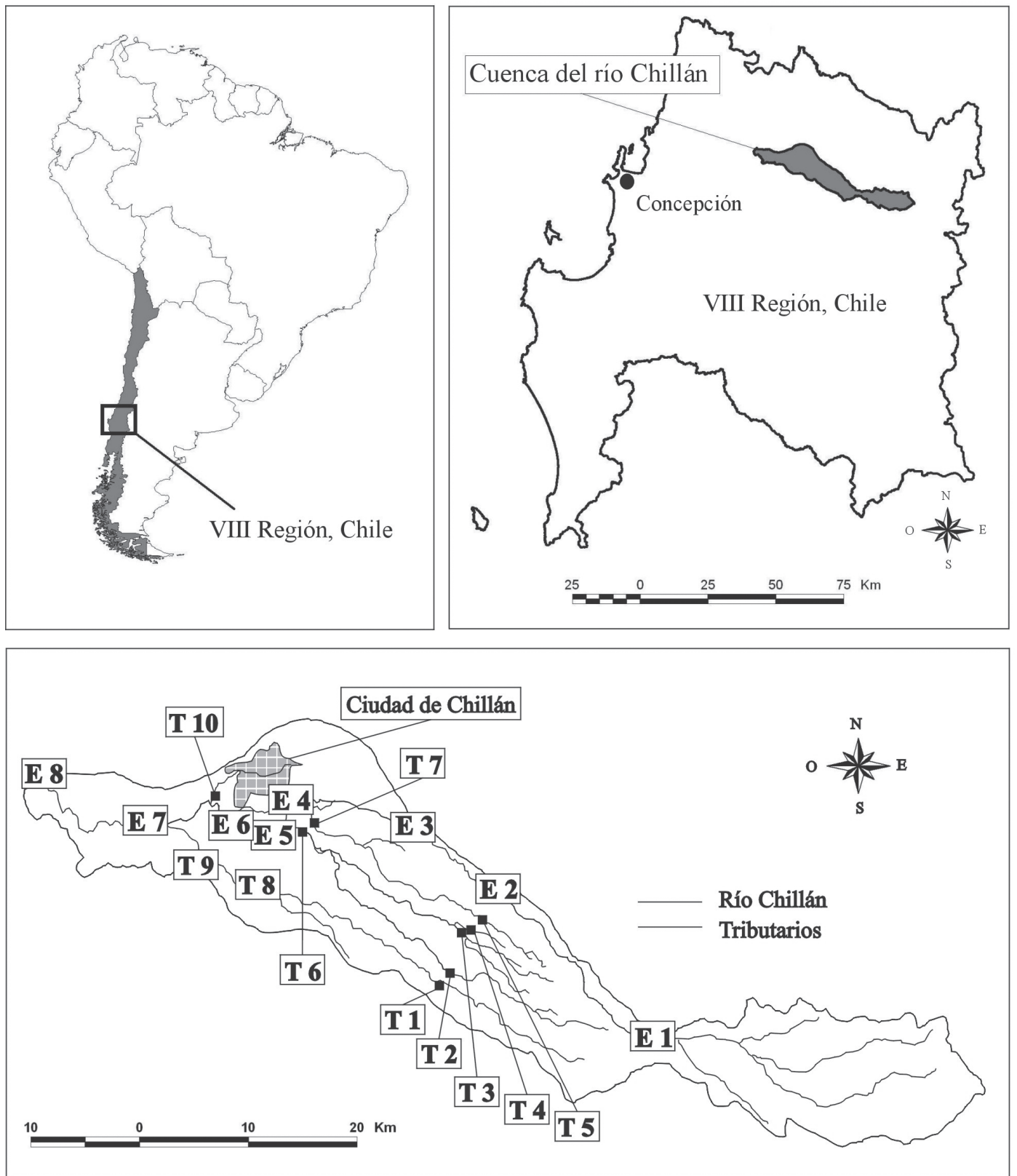

Fig. 1: Estaciones de muestreo en el río Chillán (E1-E8) y sus principales tributarios (T1-T10). Sampling stations on the Chillán River (E1-E8) and its tributaries (T1-T10). 
Obtención y análisis de las comunidades de macrozoobentos

Los muestreos fueron realizados en dos períodos de primavera y dos de verano, por ser estos donde la fauna se encuentra mejor representada (25/10/1999, 29/01/2000,3/11/2000 y 22/2/ 2001). Las muestras cuantitativas se realizaron mediante una red Surber de $0,09 \mathrm{~m}^{2}$ de área de superficie de muestreo y $250 \mu \mathrm{m}$ de abertura de malla, considerando seis réplicas por estación, estandarizando los sitios de muestreo con fondos de cantos rodados (entre 6-10 cm), con velocidades moderadas $\left(0,1-0,2 \mathrm{~m} \mathrm{~s}^{-1}\right)$ y a una profundidad entre 0,20 y $0,25 \mathrm{~m}$. Paralelamente se consideraron muestreos con sustratos artificiales correspondientes a canastos plásticos llenos de piedras de color y tamaño estandarizado (cuatro réplicas), los que fueron depositados en el lecho del río en tres ocasiones por un periodo cercano a un mes (19/1-10/2, 14/ $10-13 / 11$ de 2000 y $11 / 5-21 / 6$ de 2001), con el fin de registrar resultados comparativos con estas metodología, la cual ha sido sugerida por Alba-Tercedor (1996), cuando resulte imposible realizar muestreos directos.

Por otro lado, se realizaron muestreos cualitativos mediante el uso de redes de mano, obteniendo un catastro general de los taxa presentes, con el fin de complementar la información según lo exigen las metodologías propuestas (Prat et al. 2000). Las muestras obtenidas fueron individualizadas, para la posterior separación e identificación de los organismos bajo lupa estereoscópica (Zeiss Stemi SR) y con el apoyo de literatura especializada, tratando de llegar hasta el nivel taxonómico más bajo posible, siguiendo principalmente a: CSIRO (1991), Domínguez et al. (1992), Holzenthal \& Harris (1992), Castellanos (1994), Hauer \& Lamberti (1996), McLellan et al. (1996) y Fernández \& Domínguez (2002).

Para todos los muestreo, las réplicas fueron integradas y expresadas como número de individuos por $\mathrm{m}^{2}$, obteniendo una matriz correspondiente a la abundancia de cada taxa por estación de muestreo (Miranda 1987, Torralva et al. 1996, Rieradevall et al. 1999), sobre la cual se realizó el análisis de las variables comunitarias determinando riqueza específica (s), diversidad de Shannon $\log _{2}\left(\mathrm{H}^{\prime}\right)$ y Shannon máxima (H'máx), equitatividad (J) y diversidad de Simpson(D).

\section{Índices bióticos para definir calidad del agua}

Existe una extensa lista de índices bióticos (IB), sin embargo, reconociendo que muchos de ellos son modificaciones y/o adaptaciones, este estudio contempló la aplicación de cuatro de ellos. El primero corresponde al Índice Biótico Extendido (IBE) el cual es una modificación del IB de Woodiwis (1978), adaptado a sistemas fluviales italianos por Ghetti (1986) y que desde 1997 es aplicado como normativa por todas las agencias de protección ambiental de Italia. Este mismo IB ha sido adaptado por Prat et al. (1986), para los ríos Besós y Llobregat (España), denominándolo BILL y revisado por Muñoz \& Prat (1994). La aplicación considera la identificación de familias, géneros y especies (ver Guetti 1996), los cuales son relacionados en una tabla de doble entrada (Tabla 1), donde la horizontal corresponde a las unidades sistemáticas de más a menos sensibles presentes en la muestra o sector de estudio. La entrada vertical es definida por el número total de unidades sistemáticas encontradas. De la intersección de ambas entradas se obtiene el puntaje que se relaciona a una clase de calidad ambiental y eventualmente, puede ser asociada a un color para su representación cartográfica (Tabla 2).

El segundo IB corresponde al British Biological Monitoring Working Party score system (BMWP) de Armitage et al. (1983) el cual ha sufrido una serie de revisiones y readaptaciones a sistemas fluviales de la Península Ibérica (Alba-Tercedor \& SánchezOrtega 1988, Muñoz \& Prat 1992, 1994, AlbaTercedor 1996, Prat et al. 2000, Alba-Tercedor et al. 2002). También este índice fue adaptado para algunos sistemas fluviales de Colombia (Roldán 1988) y de Australia (Tiller \& Metzelling 2002). Su aplicación consiste en identificar las familias por segmento y asignación de un valor de tolerancia (BMWP, Tabla 3). Los valores totales son sumados y se obtiene el valor final asociado a la clase de calidad (Tabla 2). Sin embargo, siguiendo el método original (Armitage et al. 1983), estos valores pueden ser divididos por el total de familias encontradas asignando un puntaje ("score") que de acuerdo a la misma Tabla 2, se corresponden a cinco clases de calidad ambiental denominadas ASPT (Average Score per Taxon, Friedich et al 1996). Esta misma 
adaptación fue hecha para Australia, ajustados los valores de tolerancia a sus aguas denominándolo SIGNAL (Stream Invertebrates Grade Number-Averange Level, Chessman 1995, 2003, Tiller \& Metzelling 2002).

La cuarta opción consideró el IB de Familias, el cual fue desarrollado por Chuter (1972) para ríos de Sudáfrica y modificada por Hilsenhoff (1988) para aguas de climas templados de Norteamérica y que actualmente es uno de los más utilizado por la USEPA (Mackie 2001), con una aplicación reciente en Chile (Figueroa et al. 2003). Este índice requiere una aproximación cuantitativa de las familias y asignación de un valor de tolerancia para cada una de ellas (IBF, Tabla 4). El resultado resulta de la razón: $I B F=$ $1 / \mathrm{N} \sum \mathrm{n}_{\mathrm{i}} \mathrm{t}_{\mathrm{i}}$, donde $\mathrm{n}_{\mathrm{i}}$ es el número de individuos en una familia; $t_{i}$ el puntaje de tolerancia de cada familia y $\mathrm{N}$ el número total de individuos en la muestra (estación), el valor obtenido igualmente es asociado a una clase de calidad (Tabla 2). Este índice originalmente considera siete clases de calidad que fueron reducidas a 5 , por medio de una ampliación de las clases en igual proporción, con el fin de hacerlo comparable a los otros IB.

\section{TABLA 1}

Tabla de doble entrada para la determinación del IBE modificada para ríos mediterráneos de Chile (ChIBE)

Double entrance table for the determination of the IBE, modified for Chilean Mediterranean rivers (ChIBE)

\begin{tabular}{|c|c|c|c|c|c|c|c|c|c|c|}
\hline \multirow{2}{*}{$\begin{array}{l}\text { Grupos faunísticos y número de } \\
\text { unidades sistemáticas por grupo }\end{array}$} & & \multicolumn{9}{|c|}{ Número total de unidades sistemáticas (US) } \\
\hline & & $0-1$ & $2-5$ & $6-10$ & $11-15$ & $16-20$ & $21-25$ & $26-30$ & $31-35$ & $36>$ \\
\hline Plecoptera & $>1 \mathrm{US}$ & - & - & 8 & 9 & 10 & 11 & 12 & 13 & 14 \\
\hline (menos Limnoperla jaffueli) & $1 \mathrm{US}$ & - & - & 7 & 8 & 9 & 10 & 11 & 12 & 13 \\
\hline Ephemeroptera más L.jaffueli & $>1 \mathrm{US}$ & - & - & 7 & 8 & 9 & 10 & 11 & 12 & - \\
\hline (menos Baetidae y Caenidae) & $1 \mathrm{US}$ & - & - & 6 & 7 & 8 & 9 & 10 & 11 & - \\
\hline Trichoptera & $>1 \mathrm{US}$ & - & 5 & 6 & 7 & 8 & 9 & 10 & 11 & - \\
\hline (más Baetidae y Caenidae) & $1 \mathrm{US}$ & - & 4 & 5 & 6 & 7 & 8 & 9 & 10 & - \\
\hline Parastacidae y/o Aeglidae & + & - & 4 & 5 & 6 & 7 & 8 & 9 & 10 & - \\
\hline Janiiridae & + & - & 3 & 4 & 5 & 6 & 7 & 8 & 9 & - \\
\hline Oligochaeta o Chironomidae & + & 1 & 2 & 3 & 4 & 5 & - & - & - & - \\
\hline Otros & + & 0 & 1 & - & - & - & - & - & - & - \\
\hline
\end{tabular}

Limnoperla jaffueli debe ser considerada con Ephemeroptera ya que es el equivalente a Leuctridae (inexistente en Chile) en el IBE. También el IBE consideraba Gammaridae, Atyidae, Paleomonidae y Asellidae que no existen en Chile. +: sin los grupos previos (tabla modificada de Guetti 1996)

Limnoperla jaffueli should be considered with Ephemeroptera since it is the equivalent of Leuctridae (nonexistent in Chile). The IBE also considers Gammaridae, Atyidae, Paleomonidae, and Asellidae, which do not exist in Chile. +: Without the previous groups (modified table from Guetti 1996)

\section{TABLA 2}

Tabla de transformación a cinco clases de calidad para los índices utilizados, su relación con las características ambientales y el color para su representación cartográfica

Transformation to five quality ranges for each of the indexes used its relationship with the environmental characteristics, and the color of its cartographic representation

\begin{tabular}{lcccllr}
\hline Clase & ChIBE & ChBMWP & ChSIGNAL & ChIBF & Características ambientales & Color \\
\hline I & $>10$ & $>100$ & $>7$ & $0,00-3,75$ & Muy bueno, no perturbado & Azul \\
II & $8-9$ & $61-100$ & $6-7$ & $3,76-4,63$ & Bueno, moderadamente perturbado & Verde \\
III & $6-7$ & $36-60$ & $5-6$ & $4,64-6,12$ & Regular, perturbado & Amarillo \\
IV & $4-5$ & $16-35$ & $4-5$ & $6,13-7,25$ & Malo, muy perturbado & Naranja \\
V & $<4$ & $<15$ & $<4$ & $7,26-10,00$ & Muy malo, fuertemente perturbado & Rojo \\
\hline
\end{tabular}




\section{TABLA 3}

Valores de tolerancia para macroinvertebrados bentónicos dulceacuícolas para ríos mediterráneos de Chile (ChBMWP y ChSIGNAL, modificado de Alba-Tercedor 1996). Las letras mayúsculas en la primera columna indican el orden y/o clase para facilitar la búsqueda en Anexo 1

Tolerance values to freshwater benthic macroinvertebrates from Chilean Mediterranean rivers (ChBMWP and ChSIGNAL, modified from Alba-Tercedor 1996). Capital letters in the first column indicate the order to facilitate searches, see Appendix 1

Familias presentes Puntuación

P Austroperlidae, Diaphipnoidae, Eustheniidae, Notonemouridae, Perlidae

E Nesameletidae, Ameletopsidae, Oligoneuriidae, Coloburiscidae

$\mathrm{T}$ Anomalopsychidae, Calamoceratidae, Helicophidae, Kokriidae, Philopotamidae, Sericostomatidae,

Stenopsychidae

D Blephariceridae

C Limnichidae, Psephenidae

E Leptophlebiidae

Glossosomatidae, Limnephilidae

Athericidae, Dixidae

Oniscigastridae

Phylorheytidae, Polycentropodidae, Tasiimidae

Calopterygidae, Libellulidae

Parastacidae

Gripopterygiidae

(d)

Lestidae, Gomphidae, Corduliidae, Coenagrionidae

Hydroptilidae

Ceratopogonidae

Petaluridae, Aeshnidae

Elmidae

$\mathrm{Cr}$ Aeglidae, Hyallelidae

M1 Ancylidae, Chilinidae, Hyriidae

Hydropsychidae

Mg Corydalidae

D Tipulidae, Simuliidae

C Dryopidae, Gyrinidae

$\mathrm{Tu}$ Turbellaria*

M1 Amnicolidae

E Caenidae

Mg Sialidae

D Tabanidae, Stratiomyidae, Empididae, Limoniidae, Psychodidae

C Haliplidae, Curculionidae, Psephenidae

$\mathrm{H}$ Belostomatidae

A Acari*

C Hydrophilidae, Dytiscidae

Gerridae, Notonectidae, Corixidae

M1 Lymnaeidae, Physidae, Planorbidae, Sphaeriidae

$\mathrm{Cr} \quad$ Janiiridae

Hi Hirudinea*

D Chironomidae, Culicidae, Ephydridae

D Syrphidae

O1 Oligochaeta

Se han eliminado aquellas que no se encuentran en Chile, así como incorporado otras asignando puntajes de tolerancia de acuerdo a datos de Hilsenhoff (1988), Bode (1988), Lennat (1993), Roldán (1999), Chessman (1995, 2003), Tiller \& Metzelling (2002), Mackie (2001), Prat et al. (2000) y Figueroa et al. (2003). Tabla modificada de Alba-Tercedor (1996); (*) se consideran todas las familias dentro del grupo

Families not found in Chile have been eliminated, whereas others have been incorporated and assigned tolerance values according to the data from Hilsenhoff (1988), Bode (1988), Lennat (1993), Roldán (1999), Chessman (1995, 2003), Tiller \& Metzelling (2002), Mackie (2001), Prat et al. (2000), and Figueroa et al. (2003). Table modified from Alba-Tercedor 1996; (*) all families within the group were considered 
Cada uno de los índices debió ser adaptado a aguas chilenas, excluyendo las familias no presentes e incluyendo aquellas de distribución anfinótica o que son propias del Neotrópico, por ello se ha antepuesto "Ch" para cada uno de ellos. Al respecto, hoy existen complejas herramientas estadísticas que permiten una aproximación multimétrica para relacionar variables físico-químicas versus valores de tolerancia (Chessman 2003), pero en Chile se carece de una base de datos adecuada para un levantamiento de este tipo, por lo cual se recurre a la información bibliográfica y acercamiento biogeográfico que mejor represente la realidad, como puede ser Australia a través de AusRivas (Tiller \& Metzelling 2002, Chessman 2003) y la experiencia de los autores. Ello explica la ausencia de algunas familias en ChIBF (Tabla 4), que si están presentes en el ChSIGNAL (Tabla 3), de donde se tiene la información.

TABLA 4

Valores de tolerancia para macroinvertebrados bentónicos dulceacuícolas para ríos mediterráneos de Chile (ChIBF)

Tolerance values for freshwater benthic macroinvertebrates from Chilean Mediterranean rivers (ChIBF)

\begin{tabular}{|c|c|c|c|c|c|}
\hline Plecoptera & & Trichoptera & & Coleoptera & \\
\hline Gripopterygiidae & 1 & Calamoceratidae & 3 & Elmidae & 4 \\
\hline Notonemouridae & 0 & Glossosomatidae & 0 & Psephenidae & 4 \\
\hline Perlidae & 1 & Helicopsychidae & 3 & & \\
\hline Diamphipnoidae & 0 & Hydropsychidae & 4 & Diptera & \\
\hline Eustheniidae & 0 & Hydroptilidae & 4 & Athericidae & 2 \\
\hline Autroperlidae & 1 & Leptoceridae & 4 & Blephariceridae & 0 \\
\hline Limnephilidae & 2 & & & Ceratopogonidae & 6 \\
\hline Ephemeroptera & & Ecnomidae & 3 & Chironomidae & 7 \\
\hline Baetidae & 4 & Helicophidae & 6 & Empididae & 6 \\
\hline Caenidae & 7 & Polycentropodidae & 3 & Ephydridae & 6 \\
\hline Leptophlebiidae & 2 & Philopotamidae & 2 & Psychodidae & 10 \\
\hline Nesameletidae & 7 & Hydrobiosidae & 0 & Simuliidae & 6 \\
\hline Oligoneuridae & 2 & Sericostomatidae & 3 & Syrphidae & 10 \\
\hline Ameletopsidae & 2 & & & Tabanidae & 6 \\
\hline \multirow[t]{2}{*}{ Oniscigastridae } & 3 & Megaloptera & & Tipulidae & 3 \\
\hline & & Corydalidae & 0 & & \\
\hline Odonata & & Sialidae & 4 & Amphipoda & \\
\hline Aeshnidae & 3 & & & Hyalellidae & 8 \\
\hline Calopterygidae & 5 & Lepidoptera & & & \\
\hline Gomphidae & 1 & Pyralidae & 5 & Isopoda & \\
\hline Lestidae & 9 & & & Janiriidae & 4 \\
\hline Libellulidae & 9 & Mollusca & & & \\
\hline Coenagrionidae & 9 & Amnicolidae & 6 & Decapoda & \\
\hline Cordulidae & 5 & Lymnaeidae & 6 & Aeglidae & 3 \\
\hline \multirow[t]{2}{*}{ Petaluridae } & 5 & Physidae & 8 & Parastacidae & 6 \\
\hline & & Sphaeridae & 8 & & \\
\hline \multirow[t]{2}{*}{ Acari* } & 4 & Chilinidae & 6 & Platyhelminthes & \\
\hline & & & & Turbellaria & 4 \\
\hline Oligochaeta* & 8 & Hirudinea* & 10 & & \\
\hline
\end{tabular}

Se han eliminado aquellas que no se encuentran en Chile, así como incorporado otras asignando valores de tolerancia siguiendo a diversos autores (Bode 1988, Hilsenhoff 1988, Lennat 1993, Roldán 1999, Mackie 2001, Prat et al. 2000, Figueroa et al. 2003); tabla modificada de Hauer \& Lamberti (1996); *: todas las familias se consideran dentro del grupo

Those not present in Chile have been removed, whereas others have been incorporated and assigned tolerance values according to diverse authors (Bode 1988, Hilsenhoff 1988, Lennat 1993, Roldán 1999, Mackie 2001, Prat et al. 2000, Figueroa et al. 2003); table modified from Hauer \& Lamberti (1996); *: all families are considered within the group 
A partir de estos resultados, se realizó un análisis comparativo entre los IB aplicados considerando tanto los muestreos directos como de sustratos artificiales. Como los datos confrontados son rangos que identifican clases de muy buena a mala calidad (cinco clases), se seleccionó la prueba de rangos de Friedman, la cual permite obtener un valor denotado como $\chi \mathrm{r}^{2}(\mathrm{gl}=\mathrm{k}-1)$, que se aproxima a $\chi^{2}$ cuando $\mathrm{k} \mathrm{y}$ $\mathrm{N}$ son grandes $(\mathrm{k}=3$ y $\mathrm{n}>9$, o $\mathrm{k}>3 \mathrm{y} \mathrm{n}>4)$. Bajo el mismo criterio, se realizó una comparación de pares confrontando los IB mediante la prueba de suma de rangos de Wilcoxon, de modo de identificar cual de ellos presentan mayor similitud, que para un $n>25$ asociado a una probabilidad $\mathrm{P}$, un alto valor de $\mathrm{z}$ indica diferencias significativas entre los pares (Siegel 1985). Asimismo, se realizó un análisis exploratorio de modo de establecer las mejores correlaciones (Pearson) entre las variables comunitarias y los valores absolutos de los IB utilizados.

\section{RESULTADOS}

\section{Composición faunística}

La riqueza faunística encontrada en el área de estudio se entrega en el Anexo 1. Se identificaron un total de 83 taxa, la mayoría de los cuales son estados inmaduros de insectos que alcanzan un $79,5 \%$ del total. Debido a la imposibilidad de identificar todos los taxa encontrados a nivel de especie, estos se tratan indistintamente (familia, género, especie) como entidades individuales. Los órdenes más diversos fueron Ephemeroptera con 16 taxa $(19,3 \%)$, Plecoptera con 13 taxa $(15,7 \%)$, Diptera con 12 taxa $(14,5 \%)$ y Trichoptera también con 12 taxa $(14,5 \%)$ que en conjunto alcanzan una representatividad del $64 \%$ del total de los taxa.

Los grupos más abundantes fueron Chironomidae indet. y Nais sp., y secundariamente Smicridea spp. En los experimentos de colonización destaca Tubifex sp. debido a que estos se concentran en la estación T10 (Las Toscas) estación que recibía el efluente urbano de la ciudad de Chillán (esta estación no fue muestreada directamente con Surber por precauciones sanitarias). También fueron importantes Simuliidae indet., Aprophila bidentata, Meridialaris spp.,
Andesiops sp. y Deceptiviosa sp., mientras que el único plecóptero fue Limnoperla jaffueli.

Los parámetros comunitarios obtenidos en el curso principal del río Chillán (Anexo 2) indican que la estación E1 (Esperanza) se encuentra en mejor condición en términos de riqueza faunística, diversidad (H') y equitatividad (J), siendo la estación que presenta las menores dominancia (D) y abundancia. Le secundan las estaciones E2, E3 y E4, mientras que las restantes se encuentran valores menores en términos de estas variables, especialmente las estaciones E7 y E8. Los principales tributarios tienen valores muy similares, destacando T1 y $\mathrm{T} 2$ como las estaciones con las mayores riquezas faunísticas y diversidades, mientras que T7, T9 y T10 tienen las menores. La estación T10 (Las Toscas, muestreada por medio de sustratos artificiales) mostró la menor representatividad en taxa y los valores más altos de dominancia (D) y abundancia.

\section{Índices bióticos}

Una visión espacial y temporal de los resultados obtenidos mediante los muestreos directos (Fig. 2), muestran en general una tendencia a la disminución de la calidad del agua a medida que se sigue el curso del río. También es posible apreciar que el ChIBE, ChBMWP y el ChSIGNAL muestran prácticamente las mismas clases de calidad, con un paso gradual de una muy buena o buena calidad en la parte alta de la cuenca, una calidad regular en la parte media de la cuenca y el empeoramiento aguas abajo y que se acentúa después de la aglomeración urbana de la ciudad de Chillán. Por otro lado, el ChIBF mostró peores clases de calidad que los índices anteriores e incluso, en la parte alta de la cuenca, donde prácticamente no reconoce aguas de buena calidad biológica, puesto que solo en una fecha de muestreo (febrero de 2001) muestra una comunidad estructurada de clase I en la estación E1, la cual había sido considerada a priori como una estación de referencia. De modo que el ChIBF, en términos medios muestra un sistema de calidad regular a malo. Comparativamente, esta misma tendencia es válida para los experimentos de colonización, aunque siempre presentaron una calidad levemente inferior que los muestreos directos para todos los IB aplicados. Por otro lado, permitieron abordar la estación T10, donde llega el efluente urbano de 
la ciudad y que se presenta de "muy mala calidad" incorporando sus aguas a la estación E7 del curso principal (también de "muy mala calidad").
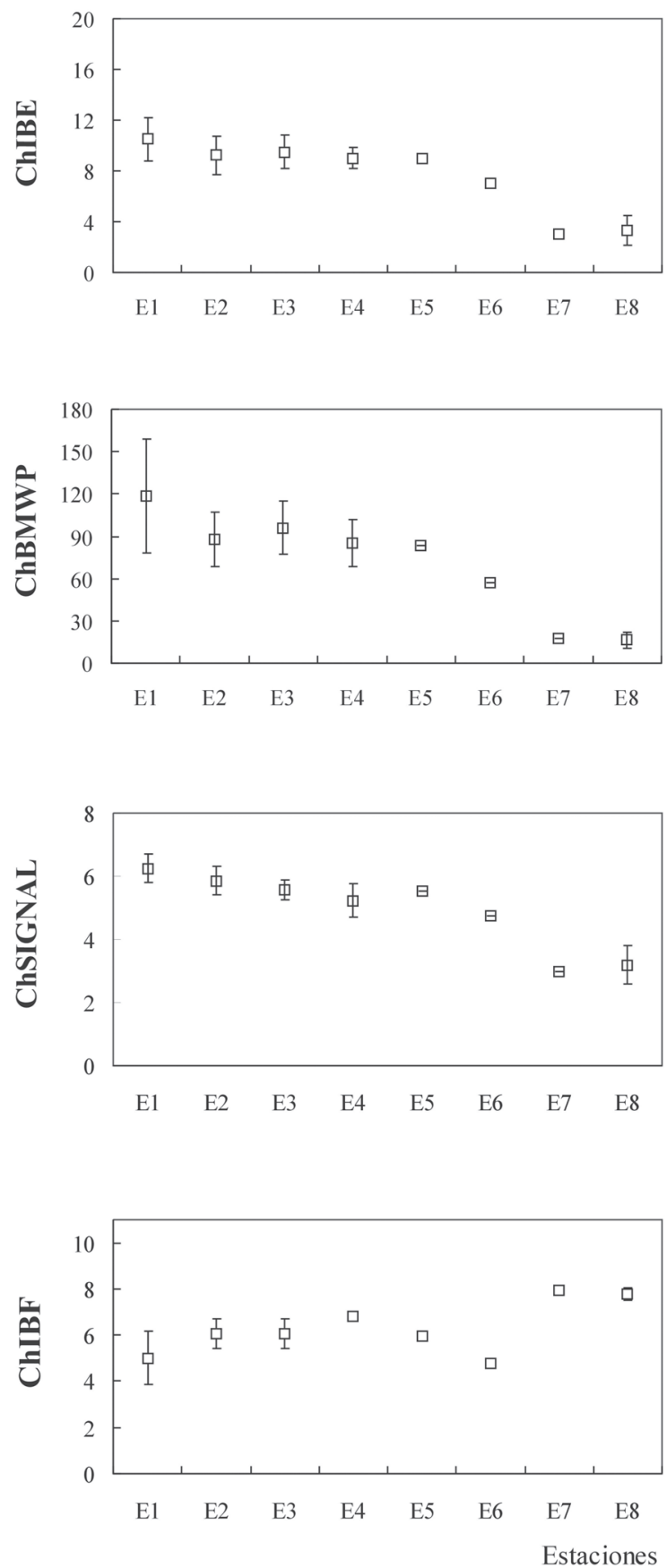

La Tabla 5 muestra el análisis comparativo entre todos los IB aplicados (prueba de rangos de Friedman), que entregó un elevado valor de $\chi \mathrm{r}^{2}$ $(\mathrm{n}=24 ; \mathrm{P}<0,001)$, por lo que las diferencias son
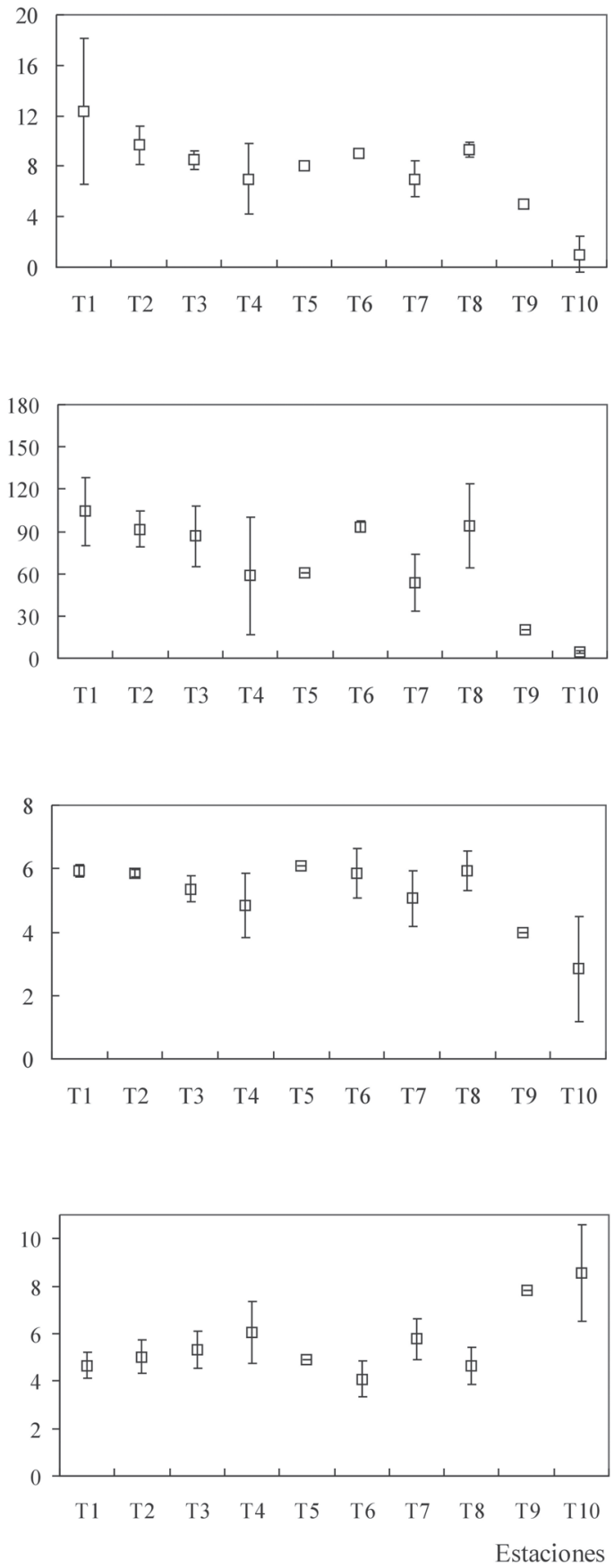

Fig. 2: Variación espacio temporal (valor medio y su desviación estándar) de los índices bióticos adaptados para Chile central (ChIBE, ChBMWP, ChSIGNAL y ChIBF), aplicados en el río Chillán (E1-E8) y sus principales tributarios (T1-T10).

Spatial and temporal variation (average and standard deviation) of the biotic indexes adapted to central Chile (ChIBE, ChBMWP, ChSIGNAL y ChIBF), applied in the Chillán River (E1-E8) and its tributaries (T1-T10). 
altamente significativas. El tratamiento de pares entre los índices (prueba de suma de rangos de Wilcoxon), identificó que los IB que presentaban mayor similitud son los pares ChIBE/ChIBMWP $(\mathrm{P}<0,009)$ y ChIBF/ChSIGNAL $(\mathrm{P}<0,001)$.

Los análisis de correlación realizados entre los IB y las variables de diversidad analizadas, son presentados en la Tabla 6, donde es posible observar que existe una alta correlación entre el ChIBE y el ChBMWP $(\mathrm{r}=0,83)$ y la riqueza específica ( $\mathrm{r}=0,81$ y 0,89 , respectivamente) y secundariamente estos tres con el ChSIGNAL $(\mathrm{r}=0,79,0,78$ y 0,61 respectivamente). Estos mismos IB no se correlacionan bien con Shannon (H') y la Equitatividad (J), para los cuales sí lo hace el ChIBF $(-0,82$ y $-0,61$, respectivamente) con valores de $\mathrm{r}$ negativos puesto que mayores valores del ChIBF indican una peor condición biológica. Por otro lado, aunque en menor grado, también se aprecia una buena correlación entre el ChSIGNAL con el ChIBE y H' (-0,66 y 0,57, respectivamente). Los resultados son consistentes puesto que en todos los casos son altamente significativos $(\mathrm{P}<0,001, \mathrm{n}=74)$.

\section{DISCUSIÓN}

La caracterización física y química de los ríos es de gran relevancia para establecer planes de manejo, aunque se reconoce su debilidad para registrar cambios temporales y de su capacidad integradora a nivel ecosistémico. Por ello hoy se aconseja complementar los estudios con la biota más representativa, donde los macroinvertebrados bentónicos son los grupos más recurrentes en este tipo de aproximación (Wrigth 1995, Bonada et al. 2006), principalmente porque representan varios

TABLA 5

Valores para las pruebas comparativas en conjunto (prueba de Friedman) y pareados (prueba de Wilcoxon) entre los índices bióticos $(*=\mathrm{P}<0,0001)$

Values for the total (Friedman test) and paired (Wilcoxon test) comparative tests of the biotic indexes $(*=\mathrm{P}<0.0001)$

\begin{tabular}{|c|c|c|c|c|}
\hline & \multicolumn{4}{|c|}{ Prueba de rangos de Friedman } \\
\hline & $\mathrm{n}$ & $\mathrm{gl}$ & Valor de $\chi \mathrm{r}^{2}$ & Valor de $\mathrm{P}$ \\
\hline \multirow[t]{3}{*}{ ChIBE / ChBMWP / ChIBF } & 74 & 3 & 100,01 & $<0,001$ \\
\hline & \multicolumn{4}{|c|}{ Prueba de la suma de rangos de Wilcoxon } \\
\hline & $\mathrm{n}$ & Valor de $\mathrm{T}$ & Valor de $\mathrm{z}$ & Valor de $\mathrm{P}$ \\
\hline ChIBE / ChIBMWP & 74 & 52,5 & 2,60 & 0,394 \\
\hline ChIBE / ChIBF & 74 & 65,0 & 5,91 & * \\
\hline ChIBE / CHSIGNAL & 74 & 18,5 & 6,04 & * \\
\hline ChIBMWP / ChIBF & 74 & 173,5 & 5,09 & $*$ \\
\hline ChBMWP / ChSIGNAL & 74 & 58,5 & 5,27 & $*$ \\
\hline ChIBF / ChSIGNAL & 74 & 224,0 & 2,32 & $*$ \\
\hline
\end{tabular}

TABLA 6

Correlaciones de Pearson obtenidas entre los índices bióticos y de diversidad.

$$
(*=\mathrm{P}<0,01 ; * *=\mathrm{P}<0,001 ; * * *=\mathrm{P}<0,0001)
$$

Pearson's correlations obtained between the biotic and diversity indexes. ( $*=\mathrm{P}<0.01, * *=\mathrm{P}<0.001 ; * * *=\mathrm{P}<0.0001)$

\begin{tabular}{lllllll}
\hline & ChIBE & ChBMWP & ChSIGNAL & ChIBF & s & H' \\
\hline ChBMWP & $0,83 * * *$ & & & & & \\
ChSignal & $0,79 * * *$ & $0,78 * * *$ & & & & \\
ChIBF & $-0,61 * * *$ & $-0,52 * * *$ & $-0,66 * * *$ & & & \\
S & $0,81 * * *$ & $0,89 * * *$ & $0,61 * * *$ & $-0,46 * * *$ & & \\
H' & $0,57 * * *$ & $0,51 * * *$ & $0,57 * * *$ & $-0,82 * * *$ & $0,51 * * *$ & $0,76 * * *$ \\
J & $0,32 *$ & $0,30 *$ & $0,29 * *$ & $-0,61 * * *$ & $0,33 *$ & \\
\hline
\end{tabular}


niveles tróficos, haciendo fluir la energía hacia niveles superiores (Wallace et al. 1997, Cummins 2002), de modo que cualquier cambio en la estructura comunitaria implica y/o explicaría cambios en toda la comunidad acuática, dando un fuerte carácter integrador del ecosistema.

El río Chillán, a pesar del grado de intervención que presenta desde su parte media, tiene una riqueza faunística importante en relación al tamaño de la cuenca $\left(757 \mathrm{~km}^{2}, 83\right.$ taxa) y en comparación con otros estudios realizados en cuencas vecinas como el río Biobío (24.400 km², 97 taxa, Arenas 1995), río Itata (11.090 km², 56 taxa; Habit et al. 1998), el río Damas $\left(514 \mathrm{~km}^{2}, 77\right.$ taxa, Figueroa et al. 2003). Sin embargo, este trabajo así como los referidos, no presentan un listado a nivel genérico o específico para todos los grupos y en muchos casos, el nivel de familia es el más utilizado. Esto señala la potencialidad de aumentar el conocimiento de la biodiversidad del lugar. Al respecto, parte de este estudio permitió conocer tres nuevas especies de Trichoptera (Holzenthal 2004) único grupo revisado por un especialista.

También ha sido posible distinguir que la mayor diversidad local se limita a la parte alta de la cuenca, tanto en el curso principal como en los tributarios, disminuyendo en la parte media y drásticamente en la parte baja de la cuenca, excepcionalmente a la altura de la ciudad de Chillán (E7 y E8). La distribución de órdenes de reconocida sensibilidad como Plecoptera, Efemeroptera y Trichoptera (Norris \& Hawkins 2000, Rabeni \& Wald 2001, Rice et al. 2001) especialmente en las estaciones E1, TI, T2, T4 y T7, indica que estas taxa no toleran las condiciones de la parte media y más baja de la cuenca, mostrando bajas abundancias cuando se encuentran.

Esto se expresa no solo para la riqueza faunística, sino también en la diversidad (H'), equitatividad (J) y dominancia (D) donde grupos oportunistas como quironómidos, oligoquetos, gastrópodos (físidos) e hirudíneos, son capaces de tolerar altas concentraciones de carga orgánica y extensos periodos con bajas concentraciones de oxígeno, facilitando altas tasas reproductiva en estos ambientes (Welch 1992, Kolar \& Frank 1993, Muñoz \& Prat 1996, Beavan et al. 2001, Kay et al. 2001, Newall \& Tiller 2002).

Los IB utilizados en este estudio mostraron una misma tendencia general y permitió definir áreas en buen estado y otras, fuertemente impactadas desde el punto de vista biológico. Sin embargo, ChIBF expresó siempre peores clases de calidad que los otros IB, mostrándose más exigente o más sensible a perturbaciones no detectadas por los otros IB, como podría ser un efecto mecánico (e.g. alteración de hábitat) o eventuales entradas de plaguicidas (contaminación difusa) asociado al uso agrícola-forestal de la cuenca. Esto podría también explicar la menor calidad biológica expresada en los muestreos de colonización, al mostrar un efecto acumulativo no detectado en un muestreo puntual y que sí detecta el ChIBF y secundariamente el ChSIGNAL.

Al respecto, debe señalarse que los índices seleccionados, son el resultado de una serie de modificaciones y revisiones en el tiempo, lo que ofrece una gran ventaja de carácter conclusivo respecto a su aplicación y rangos de tolerancia. Los cualitativos tienen un fuerte respaldo en términos de experiencia/calibración (Ghetti 2000), mientras que los cuantitativos (e.g., IBF) se fundamentan en extensas base de datos (Hinselhoff 1988) y son continuamente revisados (Lenat 1993, Lenat \& Barbour 1994, Mackie 2001). Esta situación no puede ser considerada en Chile, por la falta de experiencia en estudios de estructuras comunitarias, toxicidad, variaciones estacionales, además de las diferencias y/o semejanzas faunísticas con otras regiones del mundo de climas similares (Bonada 2003). Esto nos lleva a un proceso inevitablemente subjetivo, al no contar con un respaldo temporal que nos permita afinar las respuestas de los organismos (Rosenberg \& Resh 1993, Chapman 1995, Lowell et al. 2000).

En este sentido el ChIBF, mostró una mayor correlación con la diversidad (H'), mientras que los índices ChIBE, ChBMWP y ChSIGNAL, se correlacionaron mejor con la riqueza específica. Al respecto la diversidad (H'), tradicionalmente ha sido utilizada como un indicador de calidad ambiental (Margalef 1983). De modo que la aparición de uno o pocos individuos de una familia de baja tolerancia en un ambiente perturbado, no necesariamente lo califica de buena calidad y la suma de valores de tolerancia o la sola presencia de familias no serían buenos indicadores de la salud del sistema (Tiller \& Metzelling 2002). El ChIBF considera este concepto al tener un carácter cuantitativo y 
parcialmente el ChSIGNAL, cuando incorpora la razón entre la tolerancia y el total de las familias encontradas, factor que permitiría absorber el error de los muestreos cualitativos (Cao et al. 1997, Figueroa et al. 2005).

La cuenca del Chillán presenta aguas de excelente calidad, excepto en su porción final, donde la mayor presión la ejerce el uso urbano (Debels et al. 2005). Sin embargo, esto debe ser tratado con atención puesto que la mayor parte de la cuenca de drenaje se encuentra intervenida por agricultura intensiva y plantaciones forestales. Los suelos agrícolas suelen ser sometidos a la aplicación intermitente de plaguicidas y fertilizantes, con explotaciones anuales que dejan un mayor tiempo el suelo descubierto y removido, aumentando las probabilidades de erosión. Podraza (2002) señala que este fenómeno puede actuar como una perturbación intermedia a las perturbaciones naturales y de mayor frecuencia, la cual no permite la recolonización de especies tardías, llevándolas a su desaparición definitiva. Esto ha sido observado en este estudio al detectar una degradada calidad biológica a partir de la parte media de la cuenca.

Finalmente, este trabajo tiene carácter de propuesta, al intentar estandarizar métodos de muestreos y análisis de las comunidades de macroinvertebrados bentónicos para la evaluación de la calidad ecológica en ríos de Chile central, con miras a sustentar la futura normativa chilena para la protección de la diversidad acuática. Para ello, la utilización de ChIBF es útil por su probada sensibilidad y correlación con otras variables ambientales (Figueroa et al. 2003). Sin embargo, no es costo-efectivo al requerir un trabajo cuantitativo de mayor precisión y tiempo de análisis. El ChIBE requiere un conocimiento de la biología de las especies cuando trabaja a distintos niveles (familias, géneros y especies), mientras que el ChBMWP es de fácil aplicación pero tiene un fuerte carácter cualitativo, que lo hace muy subjetivo en sistemas pocos estudiados. Frente a esto, el ChSIGNAL corrige esta deficiencia incorporando la razón obtenida con el total de las familias encontradas. Su mayor correlación con el ChIBF y por otro lado, los valores de tolerancia de las familias fundamentado en las relaciones biogeográficas entre Chile y Australia, indican que este índice puede ser una buena herramienta para ser aplicada en Chile central, permitiendo a futuro ajustar su precisión sustentada en la experiencia y diferencias biogeográficas propias del país.

\section{AGRADECIMIENTOS}

A Elizabeth Araya y Cristina Furrianca por su apoyo de campo y laboratorio. A Roberto Urrutia y Alberto Araneda por sus valiosos comentarios y en especial, a los correctores anónimos que ayudaron a mejorar sustancialmente este trabajo. Este trabajo fue realizado en colaboración entre el Centro de Ciencias Ambientales EULA-Chile de la Universidad de Concepción y el grupo de investigación RNM-176 de la Universidad de Málaga. Financiado por el Servicio Agrícola Ganadero Proyecto SAG No VIII 4-36-0199 y parcialmente por el Proyecto DIUC 205.310.0481 de la Universidad de Concepción.

\section{LITERATURA CITADA}

ALBA-TERCEDOR J \& A SÁNCHEZ-ORTEGA (1988) Un método rápido y simple para evaluar la calidad de las aguas corrientes basado en Helawell (1978). Limnética (España) 4: 51-56

ALBA-TERCEDOR J (1996) macroinvertebrados acuáticos y calidad de las aguas de los ríos. IV Simposio del Agua en Andalucía, Almería (España) II: 203-213.

ALBA-TERCEDOR J, P JÁIMEZ-CUELLAR, M ALVAREZ, J AVILÉS, N BONADA, J CASAS, A MELLADO, M ORTEGA, I PARDO, N PRAT, M RIERADEVALL, S ROBLES, C SÁINZ-CANTERO, A SÁNCHEZORTEGA, ML SUÁREZ, M TORO, MR VIDALABARCA, S VIVAS \& ZAMORA-MUÑOZ (2002) Caracterización del estado ecológico de ríos mediterráneos ibéricos mediante el índice IBMWP (= BMWP'). Limnética (España) 21: 175-186.

ALLAN JD (2004) Landscape and Riverscapes: The influence of land use on stream ecosystems. Annual Reviews of Ecological Systems 35: 257-284

ARENAS JN (1995) Composición y distribución del macrozoobentos del curso principal del río Biobío, Chile. Medio Ambiente (Chile) 12: 39-50.

ARMITAGE PD, D MOSS, JF WRIGHT \& MT FURSE (1983) The performance of a new biological a water quality score system based on macroinvertebrates over a wide range of unpolluted running-water sites. Water Research 17: 333-347.

BARBOUR MT (1997) The re-invention of biological assessment in the U.S. Human and Ecological Risk Assessment 3: 933-940.

BEAVAN L, J SADLER \& C PINDER (2001) The invertebrate fauna of a physically modified urban river. Hydrobiologia 445: 97-108.

BONADA N (2003) Ecology of the macroinvertebrate communities in Mediterranean rivers at different scales and organization levels. Tesis de grado para optar al grado de Doctor en Ciencias. Departamento de Ecología, Facultad de Ciencias, Universidad de Barcelona. 
BONADA N, N PRAT, V RESH \& B STATZNER (2006) Developments in aquatic insect biomonitoring: a comparative analysis of recent approaches. Annual Review of Entomology 51: 495-523

BUNN AJ \& PM DAVIES (2000) Biological processes in running waters and their implications for the assessment of ecological integrity. Hydrobiologia 422/423: 461-470.

BUTCHER JT, PM STEWARD \& TP SIMON (2003) A benthic community index for streams in the northern lakes and forests ecoregion. Ecological indicators 3: 181-193.

CAMPOS H, J ARENAS, C JARA, T GONSER \& R PRINS (1984) Macrozoobentos y fauna íctica de las aguas limníticas de Chiloé y Aysén continentales (Chile). Medio Ambiente (Chile) 7: 52-64

CAO Y, A BARK \& P WILLIAMS (1997) Analyzing benthic macroinvertebrate community changes along a pollution gradient: a framework for the development of biotic indices. Water Research 31: 884-892.

CASTELLANOS ZA (1994) Fauna de agua dulce de la República Argentina. Volumen 33. Insecta: Ephemeroptera. Fascículo 1. Editorial Sigma S.R.L., Buenos Aires, Argentina. 142 pp.

CHAPMAN JC (1995) The role of ecotoxicity testing in assessing water quality. Australian Journal Ecology 20: 20-27.

CHESSMAN BC (1995) Rapid assessment of rivers using macroinvertebrates: a procedure based on habitatspecific sampling, family-level identification and a biotic index. Australian Journal Ecology 20: 122-129.

CHESSMAN BC (2003) New sensitivity grades for Australian river macroinvertebrates. Marine and Freshwater Research 54: 95-103.

CHUTTER FM (1972) An empirical biotic index of the quality of water in South African streams and rivers. Water Research 6: 19-30.

CONAMA (2004) Guía para el desarrollo de Normas para la Protección de las Aguas Continentales Superficiales. Comisión Nacional del Medio Ambiente CONAMA, Gobierno de Chile, Santiago, Chile. 23 pp.

CSIRO (1991) The insects of Australia. A textbook for students and research workers. Commonwealth Scientific and Industrial Research Organization, Division of Entomology. 1,029 pp.

CUMMINS KW (2002) Riparian-stream linkage paradigm. Verthandlundgen der internationale Vereinigung für theoretische und angewandte Limnologie 28: 49-58.

DEBELS P, R FIGUEROA, R URRUTIA, R BARRA \& X NIELL (2005) Evaluation of water quality in the Chillán river (central Chile) using physicochemical parameters and a modified water quality index. Environmental Monitoring and Assessment 110: 301-322.

DI CASTRI F (1981) Mediterranean-type shrublands of the world. En: Di Castri F, DW Goodall \& RL Specht (eds) Ecosystems of the world 11: Mediterranean-type shrublands. Elsevier, New York, USA. 643 pp.

DOMÍNGUEZ E, M HUBBARD \& W PETERS (1992) Claves para ninfas y adultos de las familias y géneros de Ephemeroptera (Insecta) Sudamericanos. Instituto de Limnología "Dr. Raúl A. Ringuelet", Biología acuática 16 (Argentina). 36 pp.

FERNÁNDEZ H \& E DOMÍNGUEZ (2002) Guía para la determinación de artrópodos bentónicos sudamericanos. Editorial Universitaria de Tucumán, Tucumán, Argentina. $282 \mathrm{pp}$

FIELD JG, KR CLARE \& RM WARWICK (1982) A practical strategy for analysing multispecies distribution patterns. Marine Ecological Progress Series 8: 37-52.

FIGUEROA R, E ARAYA \& C VALDOVINOS (2000). Deriva de macroinvertebrados bentónicos en un sector de ritrón: río Rucue, Chile centro-sur. Boletín de la Sociedad de Biología de Concepción (Chile) 71: 23-32.

FIGUEROA R, C VALDOVINOS, E ARAYA \& O PARRA (2003) Macroinvertebrados bentónicos como indicadores de calidad de agua del sur de Chile. Revista Chilena de Historia Natural 76: 275-285.

FIGUEROA R, VH RUIZ, F ENCINA-MONTOYA \& A PALMA (2005) Simplificación en el uso de macroinvertebrados en la evaluación de la calidad de las aguas en sistemas fluviales. Interciencia 30: 770-774.

FIGUEROA R, VH RUIZ, E ARAYA, X NIELL \& A PALMA (2006) Invertebrates colonization patterns in a Mediterranean Chilean stream. Hydrobiologia 571: 409-417.

GASITH A \& VH RESH (1999) Stream in Mediterranean climate region: abiotic influences and biotic responses to predictable. Annual Review of Ecology and Systematics 30: 51-81.

GHETTI PF (1986) I Macroinvertebrati nell‘Analisi di qualità dei corsi d'aqua. Stazione Sperimentale Agraria Forestale, Università di Parma, Italia. 105 pp.

GHETTI PF (2000) L'Indice Biotico Esteso (IBE). Biología Ambientale (Italia) 14: 55-61.

HABIT E, C BELTRÁN, S ARÉVALO \& P VICTORIANO (1998) Benthonic fauna of the Itata river and irrigation canals (Chile). Irrigation Science 18: 91-99

HAUER FR \& GA LAMBERTY (eds) (1996) Methods in stream ecology. Academic Press, San Diego, California, USA. 674 pp.

HINSELHOFF WL (1988) Rapid field assessment of organic pollution with a family-level biotic index. Journal of the North American Benthological Society 7: 65-68.

HOLZENTHAL RW \& SC HARRIS (1992) Hydroptilidae (Trichoptera) of Costa Rica: the genus Oxyethira Eaton. Journal of the New York Entomological Society 100: 155-177.

HOLZENTHAL RW (2004) Three new species of Chilean caddisflies (Insecta: Thichoptera). Proceedings of the Entomological Society of Washington 106: 110-117.

KARR JR \& DUDLEY DR (1981) Ecological perspectives on water quality goals. Environmental Management 5: 55-68.

KARR JR \& EW CHU (2002) Introduction: sustaining living rivers. Hydrobiologia 422/423: 1-24.

KAY WR, SA HALSE, MD SCANLON \& MJ SMITH (2001) Distribution and environmental tolerances of aquatic macroinvertebrate families in the agricultural zones of southwestern Australia. Journal of the North American Benthological Society 20: 182-199.

KOLAR CS \& JR FRANK (1993) Interaction of a biotic factor (predator presence) and abiotic factor (low oxygen) as an influence on benthic invertebrates communities. Oecologia 952: 210-219.

LENAT DR \& MT BARBOUR (1994) Using benthic macroinvertebrate community structure for rapid, cost-effective, water quality monitoring: rapid bioassessment. En: Loeb SL \& A Spacie (eds) Biological monitoring of aquatic systems: 187-215. Lewis Publishers, Ann Arbor, Michigan, USA.

LENAT DR (1993) Abiotic index for the southeastern 
United States: derivation and list of tolerance values, with criteria for assigning water-quality rating. Journal of the North American Benthological Society 12: 279-290.

LOWELL R, J CULP \& M DUBÉ (2000) A weight of evidence aproach for northern river risk assessment: integrating the effects of multiple stressors. Environmental Toxicology Chemistry 19: 11821190.

MACKIE GL (2001) Applied aquatic ecosystem concepts. Kendall/Hunt Publishing Company, Dubuque, Iowa, USA. 744 pp.

METZELING L, F WELLS \& P NEWALL (2002) The development and testing of biological objectives for the protection of rivers and streams in Victoria, Australia. Verthandlundgen der internationale Vereinigung für theoretische und angewandte. Limnologie (Germany) 28: 106-111.

MIRANDA A (1987) Utilización de macroinvertebrados bentónicos como indicadores biológicos de la calidad del agua en el río Viao-Piloña (Asturias). Limnética (España) 3: 141-150.

MUÑOZ I \& N PRAT (1992) Cambios en la calidad del agua de los ríos Llobregat y Cardener en los últimos 10 años. Tecnología del Agua (España) 91: 17-23.

MUÑOZ I \& N PRAT (1994) A comparation between different biological water quality indexes in the Llobregat Basin (NE Spain). Verthandlundgen der internationale Vereinigung für theoretische und angewandte Limnologie (Germany) 458: 1-6.

MUÑOZ I \& N PRAT (1996) Effects of water abstraction and pollution on macroinvertebrate community in mediterranean river. Limnética (España) 12: 9-16.

NEWALL P \& D TILLER (2002) Derivation of nutrients guidelines for streams in Victoria, Australia. Environmental Monitoring and Assessment 74: 85-103.

NORRIS RH \& CP HAWKINS (2000) Monitoring river health. Hidrobiología 435: 5-17.

NORRIS RH\& MC THOMS (1999) What is river health? Freshwater Biology 41: 197-209.

PLAFTKIN JL, KD BARBOUR, SK POTER, P GROSS \& RM HUGHES (1989) Rapid bioassessment protocols for use in stream and rivers. Benthic macroinvertebrates and fish. EPA/444/4-89/0001. Office of water regulations and standard, United States Environmental Protection Agency, Washington, District of Columbia, USA. 196 pp.

PODRAZA PB (2002) The intermediate-disturbance hypothesis in urban stream. Verthandlundgen der internationale Vereinigung für theoretische und angewandte Limnologie (Germany) 28: 451-454.

PRAT N, G GONZÁLEZ \& X MILLET (1986) Comparación crítica de dos índices de calidad del agua: ISQA y BILL. Artículos Técnicos (Espana) 31: 33-49.

PRAT N, A MUNNÉ, M RIERADEVALL, C SOLÀ \& N BONADA (2000) ECOSTRIMED Protocol per determinar lèstat ecològic dels rius mediterranis. Diputació de Barcelona, Àrea de Medi Ambient, Barcelona, Cataluña, Espana. 94 pp.

RABENI CF \& N WALD (2001) Bioassessment of streams using macroinvertebrates: are the chironomidae necessary? Environmental Monitoring and Assessment 71: 177-185.

RESH VH, R NORRIS \& MT BARBOUR (1995) Design and implementation of rapid assessment for water resource monitoring using benthic macroinvertebrates Australian Journal of Ecology 20: 108-121.

RESH VM, MM MYERS \& MJ HANNAFORD (1996) Macroinvertebrates as biotic indicators of environmental quality. En: Hauer FR \& GA Lamberty (eds) Methods in stream ecology. Academic Press, San Diego, California, USA. 674 pp.

RICE SP, MT GREENWOOD \& CB JOYCE (2001) Tributaries sediment sources, and the longitudinal organisation of macroinvertebrate fauna along river system. Canadian Journal of Fisheries and Aquatic Sciences 58: 824-840.

RIERADEVALL M, N BONADA \& N PRAT (1999) Community structure and water quality in the Mediterranean streams of a natural park (St. Llorec del Munt, NE Spain). Limnética (España) 17: 45-56.

ROLDÁN G (1988) Los macroinvertebrados y su valor como indicadores de la calidad del agua. Ecología, Revista de la Academia Colombiana de Ciencia (Colombia) 88: 375-387.

SCHOFIELD NJ \& PE DAVIES (1996) Measuring the health of our rivers. Water (Australia) 23: 39-43

SIEGEL S (1985) Estadística no paramétrica aplicada a las ciencias de la conducta. Ediciones Trillas S.A., México, Distrito Federal, México. 344 pp.

SIMON T (2000) The use of biological criteria as a tool for water resource management. Environmental Science \& Policy 3: 43-49.

STRAHLER AN \& AH STRAHLER (1989) Geografía física. Tercera edición. Omega, Barcelona, España. $555 \mathrm{pp}$.

TILLER D \& L METZELLING (2002) Australia-wide assessment of river health: Victorian AusRivas sampling and processing manual. Monitoring river health. Initiative Technical Report 15, Commonwealth of Australia and VIC Environmental Protection Authority, Canberra, Australia. 20 pp.

TORRALVA MM, FJ OLIVA, NA UBERO-PASCAL, J MALO \& MA PUIG (1996) Efectos de la regulación sobre macroinvertebrados en el río Segura (S.E. España). Limnetica (España) 11: 49-56.

WALLACE JB, SL EGGERT, JL MEYER \& JR WEBSTER (1997) Multiple trophic levels of a forest stream linked terrestrial litter input. Science 277: 102-104

WELCH EB (1992) Ecological effects of wastewater. Applied limnology and pollutant effects. Second edition. Chapman \& Hall, Norwell, Massachussets, USA. $425 \mathrm{pp}$

WRIGTH JF (1995) Development and use of a system for predicting the macroinvertebrate fauna in flowing waters. Australian Journal of Ecology 20: 181-197.

WRIGTH JF, DW SUTCLIFE \& MT FURSE (2000) Assessing the biological quality of fresh waters: RIVPACS and other techniques. Freshwater Biological Association, Cumbria, United Kingdom. $23 \mathrm{pp}$.

WRIGTH JF, D MOSS, PD ARMITAGE \& MT FURSE (1984) A preliminary classification of running water sites in Great Britain based on macroinvertebrate species and the prediction of community type using environmental data. Freshwater Biology 14: 221-256. 


\section{ANEXO 1}

Composición de taxa recolectados en el sistema fluvial del río Chillán $(*=$ encontrados solo en muestreos cualitativos, las letras entre paréntesis indican los órdenes para facilitar su búsqueda en la Tabla 3)

Taxa composition collected in the fluvial system of the Chillán river $(*=$ found only qualitative samples, the letters between parenthesis indicate the order to facilitate searches in Table 3 )

\begin{tabular}{|c|c|c|c|}
\hline Phylum/Clase & Clase/Orden & Familia & Taxa \\
\hline Nematoda & Adenophorea & Familia indet. & Nematoda indet. \\
\hline Nematomorpha & Gordioida & Familia indet. & Gordius sp. \\
\hline Platyhelminthes & Turbellaria $(\mathrm{Tu})$ & Dugesiidae & Dugesia sp. \\
\hline \multirow[t]{4}{*}{ Annelida } & Oligochaeta $(\mathrm{Ol})$ & Naididae & Nais sp. \\
\hline & & Lumbriculiidae & Lumbriculus sp. \\
\hline & & & Tubifex sp. \\
\hline & Hirudinea (Hi) & Familia indet. & Especie indet. \\
\hline \multirow[t]{5}{*}{ Mollusca (M1) } & Prosobranchia & Chilinidae & Chilina dombeyana (Brugiere, 1789) \\
\hline & Basommatophora & Physidae & Physa chilensis Clessin, 1886 \\
\hline & & Amnicolidae & Littoridina cumingi (D’Orbigny, 1895) \\
\hline & & Ancylidae & Gundlachia gayana (D’Orbigny, 1895) \\
\hline & & Sphaeriidae & Pisidium chilense (D’Orbigny, 1846) \\
\hline \multirow[t]{3}{*}{ Crustacea $(\mathrm{Cr})$} & Amphipoda & Hyalellidae & Hyalella sp. \\
\hline & Decapada & Parastacidae & Samastacus spinifrons (Philippi, 1882) \\
\hline & & Aeglidae & Aegla pewenchae Jara 1994 \\
\hline Chelicerata & Hydracarina (A) & Familia indet. & Especie indet. \\
\hline \multirow[t]{22}{*}{ Insecta } & Odonata $(\mathrm{O})$ & Aeshnidae & Especie indet. \\
\hline & & Gomphidae & Especie indet. \\
\hline & & Coenagrionidae & Especie indet. \\
\hline & Ephemeroptera (E) & Leptophlebiidae & Hapsiphlebia sp. \\
\hline & & & Penaphlebia sp. \\
\hline & & & Meridialaris spp. \\
\hline & & & Massartelopsis irarrazavali Demoulin, 1955 \\
\hline & & & Masartella sp.* \\
\hline & & & Nousia sp. \\
\hline & & & Demoulinellus sp. \\
\hline & & Baetidae & Deceptiviosa sp. \\
\hline & & & Andesiops sp. \\
\hline & & & Camelobaetidius sp. \\
\hline & & Amelotopsidae & Especie indet. \\
\hline & & & Chiloporter sp. \\
\hline & & Oniscigastridae & Especie indet. \\
\hline & & & Siphlonella sp. \\
\hline & & Caenidae & Caenis sp. \\
\hline & & Nesameletidae & Metamonius sp. \\
\hline & Plecoptera $(\mathrm{P})$ & Austroperlidae & Klapopterix armillata Illies, 1963 \\
\hline & & Gripopterygidae & Limnoperla jaffueli (Návas) 1928 \\
\hline & & & Antarctoperla michaelseni (Klapalek) 1904 \\
\hline
\end{tabular}


ANEXO 1 (continuación)

\begin{tabular}{|c|c|c|c|}
\hline Phylum/Clase & Clase/Orden & Familia & Taxa \\
\hline & & & Notoperlopsis femina Illies 1963 \\
\hline & & & Araucanioperla sp. \\
\hline & & & Pelurgoperla personata Illies 1963 \\
\hline & & & Senzilloides sp.* \\
\hline & & & Especie indet. \\
\hline & & Diamphipnoidae & Diamphipnopsis samali Illies 1960 \\
\hline & & & Especie indet. \\
\hline & & Perlidae & Kemnyella genualis Illies 1964 \\
\hline & & Notonemouridae & Austronemoura sp. \\
\hline & & & Udamocercia sp. \\
\hline & Hemiptera $(\mathrm{H})$ & Gerridae & Especie indet. \\
\hline & Megaloptera (Mg) & Corydalidae & Protochauliodes sp. \\
\hline & & Sialidae & Sialis chilensis McLachlan, 1870 \\
\hline & Trichoptera $(\mathrm{T})$ & Hydropsychidae & Smicridea spp. \\
\hline & & Limnephilidae & Especie indet. \\
\hline & & Leptoceridae & Nectopsyche sp. \\
\hline & & & Brachysetodes sp. \\
\hline & & & Hudsonema flaminii (Navas) 1926 \\
\hline & & & Triplectides chilensis Holzenthal, 1988 \\
\hline & & & Especie indet. \\
\hline & & Hydrobiosidae & Especie indet. \\
\hline & & Glossosomatidae & Mastigoptila brevicornuta Schmid, 1958 \\
\hline & & Hydroptilidae & Oxyethira sp. \\
\hline & & & Metrichia sp. \\
\hline & & Sericostomatidae & Parasericostoma sp. \\
\hline & Coleoptera (C) & Curculionidae & Especie indet. \\
\hline & & Elmidae & Especie indet. \\
\hline & & Gyrinidae & Especie indet. \\
\hline & & Hydrophilidae & Especie indet. \\
\hline & & Limnichidae & Especie indet. \\
\hline & & Psephenidae & Tychepsephenus felix Waterhouse 1876 \\
\hline & Diptera (D) & Chironomidae & Especie indet. \\
\hline & & Ceratopogonidae & Especie indet. \\
\hline & & Athericidae & Especie indet. \\
\hline & & Blephariceridae & Especie indet. \\
\hline & & Simuliidae & Simulium sp. \\
\hline & & Empididae & Especie indet. \\
\hline & & Ephrydidae & Especie indet. \\
\hline & & Tipulidae & Aprophila bidentata Alexander \\
\hline & & Limoniidae & Limoniinae indet. \\
\hline & & Tipulidae & Especie indet. \\
\hline & & Dixidae & Especie indet. \\
\hline & & Psychodidae & Especie indet. \\
\hline & Lepidoptera & Pyralidae & Especie indet. \\
\hline
\end{tabular}




\section{ANEXO 2}

Parámetros comunitarios $\left(\mathrm{m}^{2}\right)$ obtenidos en las estaciones del Sistema Fluvial del Río Chillán a través de muestreo con "Surber" y sustratos artificiales; $\mathrm{s}=$ riqueza específica, $\mathrm{H}$ ' = diversidad de Shannon $(\log 2)$, H'máx = diversidad de Shannon máxima, J' = equidad, D = diversidad de Simpson, $\mathrm{A}=$ abundancia

Communitarian parameters $\left(\mathrm{m}^{2}\right)$ obtained in the fluvial system stations of the Chillán river through sampling with Surber and artificial substrata; s = richness, H': Shannon's diversity $(\log 2)$, H'máx = Shannon maximum diversity, J'= evenness, $\mathrm{D}=$ Simpson's diversity, $\mathrm{A}=$ abundance

\begin{tabular}{|c|c|c|c|c|c|c|c|c|c|c|c|}
\hline Fecha & Estación & ChIBE & ChIBMWP & ChIBF & ChSIGNAL & $\mathrm{s}$ & $\mathrm{H}^{\prime}$ & H'max & $\mathrm{J}$ & $\mathrm{D}$ & A \\
\hline \multirow[t]{6}{*}{ Octubre 1999} & E1 & 11 & 144 & 4,96 & 5,76 & 31 & 2,92 & 4,95 & 0,59 & 0,25 & 3658 \\
\hline & E2 & 8 & 81 & 6,61 & 5,40 & 16 & 0,93 & 4,00 & 0,23 & 0,76 & 6992 \\
\hline & E3 & 10 & 121 & 6,43 & 5,50 & 26 & 1,10 & 4,70 & 0,24 & 0,70 & 14612 \\
\hline & E4 & 10 & 110 & 6,83 & 5,00 & 24 & 0,92 & 4,59 & 0,20 & 0,76 & 18184 \\
\hline & E7 & 3 & 18 & 7,96 & 3,00 & 6 & 0,23 & 2,81 & 0,08 & 0,93 & 358720 \\
\hline & E8 & 2 & 10 & 7,95 & 2,50 & 3 & 0,27 & 2,00 & 0,13 & 0,91 & 546978 \\
\hline \multirow[t]{10}{*}{ Enero 2000} & E1 & 11 & 117 & 5,81 & 6,16 & 22 & 2,28 & 4,46 & 0,51 & 0,26 & 36663 \\
\hline & E2 & 10 & 103 & 5,86 & 5,72 & 21 & 2,43 & 4,39 & 0,55 & 0,27 & 9548 \\
\hline & E3 & 9 & 83 & 6,22 & 5,19 & 19 & 2,02 & 4,32 & 0,47 & 0,42 & 17420 \\
\hline & E4 & 9 & 79 & 7,05 & 4,94 & 19 & 1,56 & 4,25 & 0,37 & 0,45 & 60383 \\
\hline & E8 & 4 & 22 & 7,48 & 3,67 & 7 & 1,40 & 2,81 & 0,50 & 0,44 & 5088 \\
\hline & $\mathrm{T} 1$ & 19 & 128 & 4,02 & 6,10 & 25 & 3,04 & 4,64 & 0,65 & 0,16 & 10010 \\
\hline & $\mathrm{T} 2$ & 11 & 104 & 5,43 & 5,78 & 26 & 2,88 & 4,70 & 0,61 & 0,21 & 10043 \\
\hline & $\mathrm{T} 3$ & 8 & 71 & 4,78 & 5,07 & 16 & 2,84 & 4,00 & 0,71 & 0,18 & 2922 \\
\hline & $\mathrm{T} 8$ & 9 & 90 & 5,46 & 5,29 & 20 & 2,80 & 4,32 & 0,65 & 0,18 & 18054 \\
\hline & T9 & 5 & 20 & 7,83 & 4,00 & 5 & 0,71 & 2,32 & 0,31 & 0,73 & 3611 \\
\hline \multirow[t]{12}{*}{ Noviembre 2000} & E1 & 8 & 62 & 5,86 & 6,20 & 11 & 1,79 & 3,46 & 0,52 & 0,46 & 1060 \\
\hline & E2 & 8 & 64 & 6,54 & 5,82 & 13 & 0,86 & 3,70 & 0,23 & 0,77 & 5954 \\
\hline & E3 & 8 & 80 & 6,53 & 5,71 & 15 & 1,10 & 3,91 & 0,28 & 0,69 & 14266 \\
\hline & E4 & 8 & 72 & 6,87 & 6,00 & 13 & 0,39 & 3,70 & 0,11 & 0,92 & 15541 \\
\hline & E8 & 4 & 17 & 7,96 & 3,40 & 5 & 0,23 & 2,32 & 0,10 & 0,93 & 277955 \\
\hline & $\mathrm{T} 1$ & 9 & 80 & 4,87 & 5,71 & 16 & 3,00 & 4,00 & 0,75 & 0,19 & 958 \\
\hline & $\mathrm{T} 2$ & 8 & 78 & 5,41 & 6,00 & 14 & 2,08 & 3,81 & 0,55 & 0,40 & 1230 \\
\hline & $\mathrm{T} 4$ & 5 & 29 & 6,95 & 4,14 & 8 & 0,50 & 3,00 & 0,17 & 0,88 & 1332 \\
\hline & T5 & 8 & 61 & 4,90 & 6,10 & 13 & 2,71 & 3,70 & 0,73 & 0,18 & 2506 \\
\hline & T6 & 9 & 96 & 3,55 & 6,40 & 18 & 3,15 & 4,17 & 0,76 & 0,15 & 1633 \\
\hline & $\mathrm{T} 7$ & 8 & 68 & 6,38 & 5,67 & 13 & 0,93 & 3,70 & 0,25 & 0,66 & 18070 \\
\hline & $\mathrm{T} 8$ & 9 & 66 & 3,96 & 6,00 & 16 & 2,45 & 4,00 & 0,61 & 0,30 & 1321 \\
\hline \multirow[t]{7}{*}{ Febrero 2001} & E1 & 12 & 151 & 3,41 & 6,86 & 28 & 2,67 & 4,81 & 0,55 & 0,21 & 14930 \\
\hline & E2 & 11 & 104 & 5,21 & 6,50 & 24 & 2,48 & 4,59 & 0,54 & 0,30 & 4643 \\
\hline & E3 & 11 & 100 & 5,10 & 5,88 & 25 & 2,38 & 4,64 & 0,51 & 0,28 & 11509 \\
\hline & E4 & 9 & 80 & 6,60 & 5,00 & 22 & 1,22 & 4,46 & 0,27 & 0,69 & 4551 \\
\hline & E5 & 9 & 83 & 5,93 & 5,53 & 17 & 1,37 & 4,09 & 0,34 & 0,50 & 6921 \\
\hline & E6 & 7 & 57 & 4,78 & 4,75 & 12 & 1,63 & 3,59 & 0,46 & 0,44 & 4545 \\
\hline & $\mathrm{T} 1$ & 9 & 102 & 5,11 & 6,00 & 21 & 2,59 & 4,39 & 0,59 & 0,26 & 1782 \\
\hline
\end{tabular}


ANEXO 2 (continuación)

\begin{tabular}{cccccccccccc}
\hline Fecha & Estación & ChIBE & ChIBMWP & ChIBF & ChSIGNAL & s & H' & H'max & J & D & A \\
\hline T2 & 10 & 87 & 4,21 & 5,80 & 18 & 2,68 & 4,17 & 0,64 & 0,21 & 3639 \\
T3 & 9 & 96 & 5,89 & 5,65 & 20 & 2,34 & 4,32 & 0,54 & 0,36 & 3533 \\
T4 & 9 & 78 & 5,12 & 5,57 & 16 & 2,70 & 4,00 & 0,68 & 0,21 & 2435 \\
T6 & 9 & 85 & 4,63 & 5,31 & 18 & 2,19 & 4,17 & 0,53 & 0,30 & 3724 \\
T7 & 6 & 40 & 5,15 & 4,44 & 9 & 2,03 & 3,17 & 0,64 & 0,28 & 10007 \\
T8 & 10 & 117 & 4,51 & 6,50 & 22 & 1,85 & 4,46 & 0,42 & 0,41 & 14430
\end{tabular}

Enero 19-

$\begin{array}{rrrrrrrrrrrr}\text { febrero 10, 2000 } & \text { E1 } & 8 & 41 & 6,74 & 5,13 & 9 & 1,11 & 3,17 & 0,35 & 0,67 & 2425 \\ & \text { E2 } & 9 & 63 & 6,75 & 5,73 & 20 & 0,80 & 4,32 & 0,19 & 0,8 & 25788 \\ & \text { E3 } & 8 & 82 & 6,68 & 5,47 & 17 & 1,13 & 4,09 & 0,28 & 0,69 & 23425 \\ & \text { E4 } & 7 & 64 & 6,99 & 4,57 & 16 & 1,85 & 4 & 0,46 & 0,38 & 9163 \\ & \text { E7 } & 2 & 9 & 8,00 & 2,25 & 5 & 0,54 & 2,32 & 0,23 & 0,83 & 51088 \\ & \text { E8 } & 5 & 33 & 7,06 & 4,13 & 11 & 0,42 & 3,46 & 0,12 & 0,88 & 26600 \\ & \text { T1 } & 7 & 56 & 5,94 & 5,60 & 13 & 2,32 & 3,7 & 0,63 & 0,36 & 713 \\ & \text { T2 } & 11 & 110 & 6,47 & 5,79 & 27 & 1,49 & 4,76 & 0,31 & 0,63 & 16550 \\ & \text { T3 } & 11 & 91 & 6,59 & 5,35 & 22 & 1,18 & 4,46 & 0,27 & 0,65 & 60438 \\ \text { T4 } & 6 & 42 & 6,18 & 5,25 & 9 & 1,58 & 3,17 & 0,50 & 0,52 & 1900 \\ \text { T8 } & 6 & 41 & 4,25 & 5,13 & 10 & 1,88 & 3,32 & 0,57 & 0,45 & 2225 \\ \text { T9 } & 7 & 60 & 7,00 & 5,00 & 14 & 1,53 & 3,81 & 0,40 & 0,54 & 4450 \\ \text { T10 } & 0,001 & 4 & 10,00 & 4,00 & 1 & - & - & - & 1 & 13\end{array}$

Octubre 14-

\begin{tabular}{|c|c|c|c|c|c|c|c|c|c|c|c|}
\hline \multirow[t]{12}{*}{ noviembre 13,2000} & E1 & 8 & 65 & 4,75 & 5,42 & 16 & 2,86 & 4 & 0,71 & 0,21 & 5425 \\
\hline & E2 & 8 & 55 & 6,22 & 5,50 & 13 & 1,24 & 3,7 & 0,34 & 0,65 & 10300 \\
\hline & E3 & 7 & 20 & 6,88 & 4,00 & 10 & 0,46 & 3,32 & 0,14 & 0,88 & 19350 \\
\hline & E4 & 11 & 51 & 6,61 & 5,10 & 17 & 1,27 & 4,09 & 0,31 & 0,62 & 18300 \\
\hline & E6 & 8 & 61 & 7,03 & 5,08 & 18 & 0,86 & 4,17 & 0,21 & 0,73 & 22188 \\
\hline & $\mathrm{T} 1$ & 10 & 63 & 6,34 & 5,73 & 18 & 1,83 & 4,17 & 0,44 & 0,5 & 5525 \\
\hline & $\mathrm{T} 2$ & 10 & 52 & 4,30 & 5,78 & 16 & 2,21 & 4 & 0,55 & 0,33 & 3813 \\
\hline & $\mathrm{T} 4$ & 7 & 22 & 7,16 & 4,40 & 7 & 1,79 & 2,81 & 0,64 & 0,35 & 2238 \\
\hline & $\mathrm{T} 5$ & 9 & 34 & 4,16 & 4,86 & 14 & 2,86 & 3,81 & 0,75 & 0,18 & 3175 \\
\hline & T6 & 6 & 28 & 2,97 & 5,60 & 8 & 2,86 & 3 & 0,95 & 0,15 & 975 \\
\hline & $\mathrm{T} 7$ & 5 & 13 & 7,16 & 4,33 & 5 & 0,91 & 2,32 & 0,39 & 0,63 & 2350 \\
\hline & $\mathrm{T} 8$ & 7 & 29 & 5,13 & 4,83 & 8 & 2,33 & 3 & 0,78 & 0,25 & 600 \\
\hline \multirow{9}{*}{$\begin{array}{l}\text { Mayo 11- } \\
\text { junio 26, } 2001\end{array}$} & & & & & & & & & & & \\
\hline & $\mathrm{T} 1$ & 9 & 46 & 5,43 & 5,11 & 16 & 2,28 & 3,46 & 0,66 & 0,27 & 2550 \\
\hline & $\mathrm{T} 2$ & 9 & 89 & 5,85 & 5,56 & 20 & 2,95 & 4,17 & 0,71 & 0,2 & 3175 \\
\hline & $\mathrm{T} 3$ & 7 & 31 & 7,19 & 4,43 & 16 & 2,20 & 3,32 & 0,66 & 0,29 & 1550 \\
\hline & $\mathrm{T} 4$ & 2 & 7 & 7,39 & 2,33 & 14 & 1,58 & 2 & 0,79 & 0,38 & 712,5 \\
\hline & $\mathrm{T} 5$ & 8 & 36 & 6,33 & 4,00 & 18 & 1,38 & 3,59 & 0,38 & 0,58 & 4775 \\
\hline & $\mathrm{T} 7$ & 6 & 26 & 6,60 & 4,33 & 14 & 2,02 & 2,81 & 0,72 & 0,34 & 1025 \\
\hline & T9 & 6 & 36 & 6,85 & 4,50 & 15 & 1,60 & 3,32 & 0,48 & 0,5 & 3250 \\
\hline & $\mathrm{T} 10$ & 2 & 5 & 7,13 & 1,67 & 13 & 0,59 & 2,32 & 0,26 & 0,77 & 23025 \\
\hline
\end{tabular}

